\title{
Current and future treatments for Alzheimer's disease
}

\author{
Konstantina G. Yiannopoulou and Sokratis G. Papageorgiou
}

\begin{abstract}
Alzheimer's dementia (AD) is increasingly being recognized as one of the most important medical and social problems in older people in industrialized and nonindustrialized nations. To date, only symptomatic treatments exist for this disease, all trying to counterbalance the neurotransmitter disturbance. Three cholinesterase inhibitors (Cls) are currently available and have been approved for the treatment of mild to moderate AD. A further therapeutic option available for moderate to severe AD is memantine, an N-methyl$\mathrm{D}$-aspartate receptor noncompetitive antagonist. Treatments capable of stopping or at least effectively modifying the course of $A D$, referred to as 'disease-modifying' drugs, are still under extensive research. To block the progression of the disease they have to interfere with the pathogenic steps responsible for the clinical symptoms, including the deposition of extracellular amyloid $\beta$ plaques and intracellular neurofibrillary tangle formation, inflammation, oxidative damage, iron deregulation and cholesterol metabolism. In this review we discuss current symptomatic treatments and new potential disease-modifying therapies for $A D$ that are currently being studied in phase I-III trials.
\end{abstract}

Keywords: Alzheimer's disease, amyloid, disease-modifying drugs, inflammation, tau protein, therapeutic targets

\section{Introduction}

Dementia is increasingly being recognized as one of the most important medical problems in older people with a prevalence rising from $1 \%$ at the age of 60 to at least $35 \%$ at the age of 90 [Ferri et al. 2005]. Within the spectrum of dementias, Alzheimer's disease (AD) is the most prevalent subtype, accounting for about $60 \%$ of all dementias. It is characterized clinically by progressive memory and orientation loss and other cognitive deficits, including impaired judgment and decision making, apraxia and language disturbances. These are typically accompanied by various neuropsychiatric symptoms (i.e. depression, apathy, anxiety, agitation, delusions, hallucinations). The continuing expansion of life expectancy, leading to a fast growing number of patients with dementia, particularly $\mathrm{AD}$, has led to an enormous increase in research focused on the discovery of drugs for primary, secondary or tertiary prevention of the disease. Despite all scientific efforts, at the moment there are no effective pharmacotherapeutic options for prevention and treatment of AD.

To date, established treatments are only symptomatic in nature, trying to counterbalance the neurotransmitter disturbance of the disease. Three cholinesterase inhibitors (CIs) are approved for the treatment of mild to moderate $\mathrm{AD}$ [Birks, 2006]. A further therapeutic option available for moderate to severe $\mathrm{AD}$ is memantine [McShane et al. 2006]. At the same time antipsychotic and antidepressant treatments are used for the behavioral symptoms of the disease [Ballard and Corbett, 2010].

Treatments under research include compounds that act on the pathological substrate of the disease: extracellular amyloid $\beta(\mathrm{A} \beta)$ plaques and intracellular neurofibrillary tangles (NFTs).

In this review, current symptomatic treatments and new potential disease-modifying therapies for
Ther Adv Neurol Disord

(2013) 6(1) 19-33

DOI: $10.1177 /$

1756285612461679

(c) The Author(s), 2012. Reprints and permissions: http://www.sagepub.co.uk/ journalsPermissions.nav
Correspondence to: Konstantina G. Yiannopoulou, MD Neurological Department, Laiko General Hospital of Athens, 15127Ag. Thoma

17, Athens, Greece ekati2dotenet.gr Sokratis G. Papageorgiou, PhD, MD 2nd Neurological

Department, University of Athens, University Hospital 'Attikon', Haidari, Athens, Greece 
AD that are currently being studied in phase I-III trials are discussed.

\section{Current symptomatic approaches to Alzheimer's disease}

\section{Cholinesterase inhibitors}

The cholinergic hypothesis of AD concludes that cholinergic systems in the basal forebrain are affected early in the disease process, including loss of acetylcholine neurons, loss of enzymatic function for acetylcholine synthesis and degradation, resulting in memory loss and deterioration of other cognitive and noncognitive functions such as neuropsychiatric symptoms [Bartus et al. 1982; Cummings and Back, 1998]. A strategy to enhance the cholinergic transmission by using CIs to delay the degradation of acetylcholine between the synaptic cleft has been proposed. To date, three CIs are approved for the treatment of mild to moderate AD: donepezil (Pfizer, New York, NY, USA), rivastigmine (Novartis, Basel, Switzerland) and galantamine (Janssen, Beerse, Belgium) [Farlow, 2002]. These drugs have been regarded as the standard and first-line treatment for AD. Systemic reviews including many double-blind, randomized, placebo-controlled trials (RCTs) of these three CIs all showed benefit on cognitive functions, activities of daily living (ADL), and global function for patients with mild to moderate $\mathrm{AD}$; there was no significant difference of efficacy between individual CIs [Farlow, 2002; Birks, 2006]. In addition, donepezil is now also approved for the treatment of severe AD in the USA [Cummings et al. 2010]. Although tacrine (First Horizon Pharmaceuticals, Alpharetta, Georgia, USA) was the first CI drug approved for AD in 1993, it is no longer used due to hepatotoxicity [Alfirevic et al. 2007]. Related systemic reviews showed that the incidence of gastrointestinal adverse effects, such as nausea, vomiting, diarrhea and abdominal cramp, was lower with donepezil than with rivastigmine and galantamine [Alva and Cummings, 2008]. The incidence of adverse effects was associated with higher therapeutic dose. However, it may be that galantamine and rivastigmine may be equal to donepezil in tolerability if a careful and gradual titration routine of more than 3 months is used. The dermal form of rivastigmine provides a lower dose with fewer adverse effects but comparable efficacy, and is was preferred by some caregivers [Blesa et al. 2007]. Use of CIs is also reported to be associated with increased rates of syncope, bradycardia and pacemaker insertion. The risk of these adverse events must be weighed carefully against the drugs' benefits [Gill et al. 2009].

Reviews and meta-analyses on CIs that have recently been published showed that they delay the decline in cognitive function as measured by the AD Assessment Scale - cognitive subscale (ADAS-cog), global clinical rating, behavior and ADL over 6-12-month periods. These benefits seem to be applicable to mild, moderate and severe AD [Birks, 2006; Hansen et al 2008; Qaseem et al. 2008]. Compared with those on placebo treatment, patients on CIs generally show an initial mild improvement in cognitive functions over the first 3 months. Thereafter, the mean decline in cognitive functions was also less rapid over the subsequent 3-9 months. At 6 months, the cognitive improvement (versus placebo) was 2.7 points over the Mid range of ADAS-cog [Birks, 2006; Hansen et al. 2008]. Symptoms that were improved included attention, thinking, memory, praxis, language comprehension and communication [Qaseem et al. 2008].

Initiation of CI treatment in the early stages of $\mathrm{AD}$ is preferred. A 52-week study of the efficacy of rivastigmine in patients with mild to moderately severe $\mathrm{AD}$ reported that patients with $\mathrm{AD}$ who started the CI 6 months later achieved lower cognitive performance than those who started the drug immediately after the diagnosis [Farlow et al. 2000]. Preserved cognitive function was also observed after 12 months of treatment with rivastigmine in patients with mild AD in comparison to untreated patients who markedly worsened in cognition during the same period [Almkvist et al. 2004].

\section{$N$-methyl-D-aspartate antagonist}

A further therapeutic option for moderate to severe $\mathrm{AD}$ is memantine (Lundbeck, Valby, Denmark). This drug is an uncompetitive, moderate-affinity N-methyl-D-aspartate (NMDA) antagonist believed to protect neurons from excitotoxicity. A systemic review of double-blind, parallel-group, RCT studies of memantine showed improvement in cognition, ADL and behaviors in people with moderate to severe $\mathrm{AD}$ after 6 months of use [McShane et al. 2006]. Another systemic review which included six RCT studies indicated that memantine may reduce behavioral and psychological symptoms of dementia [Maidment 
et al. 2008]. The most frequently reported adverse events in memantine trials were dizziness, headache and confusion. A small group of patients might develop agitation [Alva and Cummings, 2008].

\section{Combination therapy}

RCT studies on parallel groups of patients with moderate to severe $\mathrm{AD}$ showed a significant benefit in cognitive function, language, ADL, behaviors and global state from combination use of memantine and donepezil over the placebo group (memantine and placebo) [Tariot et al. 2004; Feldman et al.2006; Howard et al. 2012]. However, such benefit was not demonstrated in patients with mild to moderate $\mathrm{AD}$ [Farlow et al. 2010].

\section{Treatment of behavioral and psychological symptoms of dementia in Alzheimer's disease} Noncognitive neuropsychiatric symptoms or behavioral and psychological symptoms of dementia (BPSD) are common in all clinical stages of $\mathrm{AD}$ and even in amnestic mild cognitive impairment (MCI) (the predementia stage of $\mathrm{AD}$ ) with increasing prevalence when dementia progresses. They are the main determining factors for increased caregiver burden and institutionalization of patients. According to a large observational study, BPSD may be grouped into four major symptom clusters with high prevalence: psychosis (38\% of the patients, e.g. delusions), affective symptoms $(59 \%$, anxiety and depression), hyperactivity (64\%, e.g. aggression, disinhibition) and apathy (65\%) [Zec and Burkett, 2008].

CIs and memantine may have an effect on behavioral symptoms [Farlow, 2002; Birks, 2006; Maidment et al. 2008]. However, when BPSD become more severe, these antidementia drugs may not be as effective and other drugs also need to be given.

Serotonin reuptake inhibitors (SSRIs: fluoxetine, sertraline, paroxetine, citalopram, fluvoxamine) are largely considered to be among the most efficient antidepressants to treat comorbid depression in AD dementia [Zec and Burkett, 2008].

Mirtazapine, venlafaxine and duloxetine, which are combined selective noradrenalin and serotonin inhibitors (SNRIs), and bupropion are other widely used antidepressants in this population.
A few RCTs with limited numbers of patients as well as meta-analyses support their efficacy to treat depression in AD dementia [Ballard and Corbett, 2010]. SSRIs may also be taken into consideration for the treatment of agitation and psychosis in $\mathrm{AD}$ dementia [Zec and Burkett, 2008]. However, a recent randomized, multicenter, double-blind, placebo-controlled trial of sertraline or mirtazapine for depression in dementia (HTA-SADD) showed absence of benefit compared with placebo and increased risk of adverse events. The trial concluded that the current practice of using these antidepressants, with usual care, for first-line treatment of depression in Alzheimer's disease should be reconsidered [Banerjee et al. 2011].

Psychotic symptoms and agitation/aggression are commonly treated with antipsychotics in patients with $\mathrm{AD}$ dementia. Atypical agents (olanzapine, risperidone, quetiapine, ziprasidone and aripiprazole) are preferred due to their milder parkinsonian effects [Ballard and Corbett, 2010]. The use of antipsychotics has been discussed controversially, as cerebrovascular morbidity and higher mortality have been found in patients with dementia taking antipsychotics. Furthermore, the use of antipsychotics may be associated with a higher risk of hip fracture and pneumonia, as well as worsening cognitive impairment. The increased mortality may be reduced if antipsychotics are only given over a short period, as stopping the antipsychotic medication may not be associated with a subsequent increase in BPSD [Zec and Burkett, 2008].

Benzodiazepines are used to reduce agitation and anxiety. However, they can also trigger further agitation in older people. An association of greater benzodiazepine use with more rapid cognitive and functional decline has been reported in $\mathrm{AD}$ and indeed in older people in general [Zec and Burkett, 2008].

Anticonvulsant drugs like carvamazepine can also reduce $\mathrm{BPSD}$ in $\mathrm{AD}$ to some degree [Ballard et al. 2009].

It is obvious that drugs currently used for the treatment of $\mathrm{AD}$ have weak beneficial effects on cognitive function or offer some relief of BPSD. The discovery of new drugs that act during the early stages of $\mathrm{AD}$ could be considered a 'medical need' [Mancuso et al. 2011]. Early intervention is critical because a delay in treatment is associated with nonreversible symptom progression. 
The amyloid hypothesis

The primary histopathologic lesions of Alzheimer's pathology are amyloid plaques, NFTs and neuronal loss. Mature plaques consist of a central amyloid core with surrounding degenerating neurons affected by the toxic effect of the A $\beta$. NFTs consist of hyperphosphorylated tau protein that has assumed a double helical filament conformation [Cummings, 2008b].

The $A \beta$ derives from the amyloid precursor protein (APP) through sequential proteolysis by $\beta$ secretase (BACE1) in the extracellular domain and $\gamma$ secretase in the transmembrane region.

Full-length APP undergoes sequential proteolytic processing. It is first cleaved by $\alpha$ secretase (nonamyloidogenic pathway) or $\beta$ secretase (amyloidogenic pathway) within the luminal domain, resulting in the shedding of nearly the entire ectodomain and the generation of $\alpha$ or $\beta$ $\mathrm{C}$-terminal fragments (CTFs). The major neuronal $\beta$ secretase, named BACE1 ( $\beta$-site APP cleaving enzyme), is a transmembrane aspartyl protease that cleaves APP within the ectodomain, generating the $\mathrm{N}$-terminus of $\mathrm{A} \beta$. The second proteolytic event in APP processing involves intramembranous cleavage of $\alpha$ and $\beta$ CTFs by $\gamma$ secretase. Major sites of $\gamma$-secretase cleavage correspond to positions 40 and 42 of $\mathrm{A} \beta$. Amyloidogenic processing is the favored pathway of APP metabolism in neurons because of the greater abundance of BACE1, whereas the nonamyloidogenic pathway predominates in other cells [Vassar, 2004].

According to the 'amyloid hypothesis' $\mathrm{A} \beta$ production in the brain initiates a cascade of events leading to the clinical syndrome of Alzheimer's dementia [Golde, 2005]. A $\beta$ is a protein consisting of two major forms, $A \beta 40$ and $A \beta 42$. $A \beta 42$ is the most soluble form and has the tendency to aggregate into fibrils that form the major composite of amyloid plaques. It is the predominant form found in the brain parenchyma of patients with $\mathrm{AD} . \mathrm{A} \beta 40$ is mostly found in the cerebral vasculature as part of 'cerebral amyloid angiopathy'. $A \beta$ has a tendency to cluster into oligomers. Oligomers can form $\mathrm{A} \beta$-fibrils and protofibrils that will eventually form amyloid plaques, which are believed to be nontoxic. It is the forming of amyloid oligomers to which neurotoxicity is attributed and initiates the amyloid cascade. The elements of the cascade include local inflammation, oxidation, excitoxicity (excessive glutamate) and tau hyperphosphorylation. As a result of this process, tau proteins fold into intraneuronic tangles, which results in cell death. Progressive neuronal destruction leads to shortage and imbalance between various neurotransmitters (e.g. acetylcholine, dopamine, serotonin) and to the cognitive deficiencies seen in AD [Cummings, 2008a; Golde, 2005].

On the basis of findings on $\mathrm{AD}$ pathogenesis, novel treatments under development aim to interfere with the pathogenic steps previously mentioned in an attempt to block the course of the disease in its early stages [Galimberti and Scarpini, 2011; Golde, 2005]. For this reason they have been termed 'disease-modifying' drugs. In this review, possible strategies for the development of novel disease-modifying therapies will be discussed.

\section{Disease-modifying approaches to Alzheimer's disease}

The production of $A \beta$, which is a crucial step in AD pathogenesis, is the result of cleavage of APP, which is overexpressed in $\mathrm{AD}$ [Griffin, 2006]. $\mathrm{A} \beta$ forms highly insoluble and proteolysis-resistant fibrils known as senile plaques (SPs). NFTs are composed of the tau protein. In healthy subjects, tau is a component of microtubules, which are the internal support structures for the transport of nutrients, vesicles, mitochondria and chromosomes within the cell. Microtubules also stabilize growing axons necessary for the development and growth of neurons [Griffin, 2006]. In AD, tau protein is abnormally hyperphosphorylated and forms insoluble fibrils, causing deposits within the cell.

Thus, both $A \beta$ and tau are prime targets for disease-modifying therapies in AD. From this point of view, $\mathrm{AD}$ could be prevented or effectively treated by decreasing the production of $A \beta$ and tau; preventing aggregation or misfolding of these proteins; neutralizing or removing the toxic aggregate or misfolded forms of these proteins; or a combination of these modalities.

A number of additional pathogenic mechanisms have been described, possibly overlapping with $\mathrm{A} \beta$ plaques and NFT formation, including inflammation [Griffin, 2006], oxidative damage [Reddy et al. 2009], iron deregulation [Adlard and Bush, 2006] and cholesterol metabolism [Stefani and Liguri, 2009]. 
Table 1. Disease-modifying treatments: modulation of amyloid deposition.

\begin{tabular}{|c|c|c|}
\hline $\begin{array}{l}\text { Drugs interfering with } A \beta \\
\text { aggregation }\end{array}$ & Selective $A \beta 42$-lowering agents & Immunotherapy \\
\hline $\begin{array}{l}\text { Anti-amyloid aggregation agents: } \\
\text { Tramiprosate: phase III trial (-) } \\
\text { Colostrinin: phase II trial ( } \pm \text { ) } \\
\text { Scyllo-inositol: phase II trial }( \pm]\end{array}$ & $\begin{array}{l}\text { Inhibition of } \beta \text {-secretase: } \\
\text { Nonpeptidic inhibitors: trials in } \\
\text { animal models }(+) \\
\text { CTS-21166: phase I trials }(+)\end{array}$ & $\begin{array}{l}\text { Active immunization (vaccination): } \\
\text { AN-1792: phase II trial (-) } \\
\text { CAD-106, V950, ACC-001: phase II } \\
\text { trials } \\
\text { MABT5102A, PF-04360365, R1450: } \\
\text { phase I trials (+) } \\
\text { GSK933776A: phase I trials } \\
\text { DNA epitope vaccine antibodies } \\
\text { against the } \beta \text {-secretase cleavage } \\
\text { site mucosal vaccination: trials in } \\
\text { animal models (+) }\end{array}$ \\
\hline \multirow[t]{2}{*}{$\begin{array}{l}\text { Drugs interfering with metals: } \\
\text { PBT2: phase Ila trial (+) }\end{array}$} & $\begin{array}{l}\text { Inhibition of } \gamma \text {-secretase: } \\
\text { Semagacestat: phase III trials (-) } \\
\text { Tarenflurbil: phase III trials (-) } \\
\text { Avagacestat: phase II trial }\end{array}$ & $\begin{array}{l}\text { Passive immunization } \\
\text { (monoclonal antibodies): } \\
\text { Bapineuzumab: phase II trial (+) } \\
\text { Solanezumab: phase II trial } \\
\text { IVIg: phase III trial }\end{array}$ \\
\hline & $\begin{array}{l}\text { Activation of } \alpha \text {-secretase: } \\
\text { Etazolate: phase Ila trial }(+)\end{array}$ & \\
\hline
\end{tabular}

\section{Disease-modifying treatments: modulation} of amyloid deposition

Drugs interfering with amyloid $\beta$ deposition. Anti-amyloid aggregation agents The hypothesis that aggregation of $\mathrm{A} \beta$ leads to toxic oligomeres has driven research into studying compounds that could prevent this aggregation (Table 1) [Cummings, 2008b; Golde, 2005].

The only $A \beta$ aggregation inhibitor reaching phase III is the synthetic glycosaminoglycan 3-amino1-propaneosulfonic acid (3APS, tramiprosate) [Gauthier et al. 2009]. It is designed to interfere with the binding of glycosaminoglycanes and $A \beta$. Disappointing results of the North American phase III trial in the year 2007 have led to discontinuation of the European phase III trial. Nevertheless, 3APS will now be commercialized as a branded nutraceutical. However, recent data suggest that tramiprosate promotes an abnormal aggregation of the tau protein in neuronal cells [Santa-Maria et al. 2007]. These results emphasize the importance of testing the potential drugs for the treatment of AD on both types of pathology (amyloid and tau).

Another molecule undergoing testing is colostrinin, a proline-rich polypeptide complex derived from sheep colostrum (O-CLN; ReGen
Therapeutics, London, UK). Colostrinin inhibits $\mathrm{A} \beta$ aggregation and neurotoxicity in cellular assays and improves cognitive performance in animal models. Although a phase II trial demonstrated modest improvements in Mini Mental State Evaluation scores for patients with mild AD over a treatment period of 15 months, this beneficial effect was not sustained during an additional 15 months of continued treatment [Bilikiewicz and Gaus, 2004].

Another compound named scyllo-inositol is able to stabilize oligomeric aggregates of $A \beta$ and inhibit $A \beta$ toxicity in mouse hippocampus. An 18-month, randomized, double-blind, placebocontrolled, dose-ranging, safety and efficacy study of oral scyllo-inositol (ELND005) in participants with mild to moderate $\mathrm{AD}$ has been carried out by Transition Therapeutics (Toronto, ON, Canada)/Elan (Dublin, Ireland). A long-term follow-up class II study in subjects with AD provided insufficient evidence to support or refute a benefit of ELND005.

Primary clinical efficacy outcomes were not significant. The safety and cerebrospinal fluid (CSF) biomarker results will guide selection of the optimal dose for future studies, which will target earlier stages of AD [Salloway et al. 2011]. 
Drugs interfering with metals Zinc ( $\mathrm{Zn}$ ) and copper $(\mathrm{Cu})$ are both involved in the aggregation of $\mathrm{A} \beta 42$. Several chelators of $\mathrm{Zn} / \mathrm{Cu}$ have been shown to inhibit $A \beta$ aggregation in vitro and in animal studies. PBT2 is a second-generation $8-\mathrm{OH}$ quinoline metal-protein-attenuating compound that affects the $\mathrm{Cu} 2^{+}$-mediated and $\mathrm{Zn} 2^{+}-$ mediated toxic oligomerization of $\mathrm{A} \beta$. A recent phase IIa study concluded that the safety profile is favorable for the ongoing development of PBT2. The effect on putative biomarkers for $\mathrm{AD}$ in $\mathrm{CSF}$ but not in plasma suggests a central effect of the drug on $A \beta$ metabolism. Cognitive efficacy was restricted to two measures of executive function. In the post hoc analysis, the cognitive, blood marker and CSF neurochemistry outcomes from the trial were subjected to further analysis. Ranking the responses to treatment after 12 weeks with placebo, PBT2 $50 \mathrm{mg}$ and PBT2 $250 \mathrm{mg}$ revealed that the proportions of patients showing improvement were significantly greater in the PBT2 $250 \mathrm{mg}$ group than in the placebo group. These findings further encourage larger-scale testing of PBT2 for AD [Faux et al. 2010].

Selective $A \beta 42$-lowering agents. $\quad A \beta$ is generated through proteolytic processing of the transmembrane peptide APP. APP can be cleaved by two competing proteases, $\alpha$ secretase and $\beta$ secretase. Only cleavage by $\beta$ secretase, followed by $\gamma$-secretase cleavage, which in $\mathrm{AD}$ is the dominant pathway, will lead to production of $A \beta 40$ and $\mathrm{A} \beta 42$. By inhibiting $\beta$ secretase and $\gamma$ secretase or by increasing $\alpha$-secretase cleavage, $A \beta$ production may be reduced [Cummings, 2008a].

$\beta$-site AP- cleaving enzyme inhibition The $\beta$-secretase enzyme BACE1 is a promising therapeutic target, although the development of a BACE1 inhibitor therapy is problematic for two reasons. First, BACE1 has been found to have important physiological roles. Therefore, inhibition of the enzyme could have toxic consequences. Second, the active site of BACE1 is relatively large, and many of the bulky compounds that are needed to inhibit BACE1 activity are unlikely to cross the blood-brain barrier. Many of compounds able to inhibit BACE are still in the preclinical phase. Inhibitors based on the peptidomimetic strategy suffer from well known difficulties associated with polypeptides, such as blood-brain barrier crossing, poor oral bioavailability and susceptibility to P-glycoprotein transport. Efforts to overcome these problems led to the design of new nonpeptidomimetic $\beta$-secretase inhibitors that show high selectivity over BACE2 (BACE1/BACE2 selectivity >100) and other human proteases (cathD, pepsin and renin). Their weak or nonpeptidic character favors CNS penetration and oral bioavailability [Silvestri, 2009]. A ligand-based computational approach is currently used to identify the molecular chemical features required for the inhibition of BACE1 enzyme [John et al. 2011].

Only a few $\beta$-secretase inhibitors have entered clinical trials to date. The first publicly announced phase I clinical trial on a $\beta$-secretase inhibitor CTS-21166 was conducted by CoMentis (South San Francisco, USA) [Hey et al. 2008; Albert, 2008; Panza, 2009]. Phase I clinical trials on CTS-21166 have been carried out in healthy young men and evaluated for safety and preliminary $A \beta$ responses. In these clinical trials, $\beta$-secretase inhibitor has been shown to reduce human plasma $A \beta$ [Hey et al. 2008]. Clearly, the hope for the next step would be to develop inhibitors with better pharmaceutical properties and to carry out well designed efficacy trials to determine if they can rescue cognitive decline in patients with $\mathrm{AD}$ [Ghosh et al. 2012].

$\gamma$-Secretase inhibition $\gamma$ Secretase is a nucleoprotein complex with at least four different proteins from which preseniline PS- 1 and PS-2 seem to be responsible for the enzymatic action on APP. Unfortunately, besides APP, $\gamma$ secretase has many other substrates and cleaves several other transmembrane proteins, including the Notch receptor 1 , which is necessary for growth and development. Notch-related side effects of $\gamma$-secretase inhibition (severe gastrointestinal and hemopoetic side effects) have been hampering the development of clinically useful $\gamma$-secretase inhibitors so far [Wong et al. 2004].

The most studied $\gamma$-secretase inhibitor, which is semagacestat (LY-450139), was shown to dosedependently decrease the generation of $A \beta$ in the CSF of healthy people [Siemers et al. 2005]. Unfortunately, two large phase III clinical trials of semagacestat in patients with mild to moderate $\mathrm{AD}$ were prematurely interrupted because of the observation of detrimental effects on cognition and functionality in patients receiving the drug compared with those receiving placebo. These detrimental effects were mainly ascribed to the inhibition of Notch processing and to the accumulation of the neurotoxic precursor of $A \beta$ (the C-terminal fragment of APP or CTF $\beta$ ) resulting 
from the block of the $\gamma$-secretase cleavage activity on APP [Imbimbo and Giardina, 2011]. Two large phase III studies in patients with mild AD with tarenflurbil (or R-flurbiprofen), which is a putative $\gamma$-secretase modulator, were also completely negative. The failure of tarenflurbil was ascribed to low potency and brain penetration. New Notchsparing $\gamma$-secretase inhibitors and more potent and brain penetrant $\gamma$-secretase modulators are being developed with the hope of overcoming the previous setbacks [Imbimbo and Giardina, 2011].

A potent $\gamma$-secretase inhibitor, BMS-708163 (avagacestat; Bristol-Myers Squibb, New York, NY, USA), was tested in a phase I clinical trial. After 18 days, BMS-708163 caused a decrease in CSF $A \beta 40$ and $A \beta 42$ of $30 \%$ following a daily dose of $100 \mathrm{mg}$ as well as a decrease of $60 \%$ at a daily dose of $150 \mathrm{mg}$. A phase II study is ongoing [Tong et al 2012].

$\alpha$-Secretase potentiation Etazolate (EHT 0202; ExonHit Therapeutics, Paris, France) stimulates the neurotrophic $\alpha$-secretase (nonamyloidogenic) pathway and inhibits $A \beta$-induced neuronal death, providing symptomatic relief and modifying disease progression. The recent pilot, randomized, double-blind, placebo-controlled, parallel group, multicentre, phase IIA study was conducted in 159 randomized patients with mild to moderate AD. EHT0202 was shown to be safe and generally well tolerated. These first encouraging safe results support further development of EHT0202 to assess its clinical efficacy and to confirm its tolerability in a larger cohort of patients with $\mathrm{AD}$ and for a longer period of time [Vella et al. 2011].

Immunotherapy. Immunotherapy is one of the strategies being studied by most pharmaceutical companies. The mechanism behind amyloid clearance by immunotherapy has not been fully elucidated. At least six mechanisms that are not mutually exclusive are considered to elicit a humoral response: First, by direct disassembly of plaques by conformation-selective antibodies; second, by antibody-induced activation of microglial cells and phagocytosis of pathological protein deposits; third, by noncomplement-mediated phagocytosis activation of microglial cells; fourth, by neutralization of toxic soluble oligomers; fifth, by a shift in equilibrium toward efflux of specific proteins from the brain, creating a peripheral sink by clearance of circulating $A \beta$ cell-mediated immune responses; and finally, immunoglobulin M (IgM)-mediated hydrolysis. All these mechanisms may play roles depending on the specific immunotherapeutic scenario [Wisniewski and Konietzko, 2008] (Figure 2).

Both active immunization (vaccination) and passive immunization (monoclonal antibodies) are being studied. After promising preclinical results in animal studies, one of the first active vaccination trials was initiated using human $A \beta 1-42$ (AN-1792) in conjunction with a T-helper adjuvant (QS-21). Unfortunately, in 2002, the phase II vaccination trial was discontinued because of the occurrence of meningoencephalitis (6\%) [Gilman et al. 2005]. Additionally, only $19.7 \%$ of the AN-1792-treated patients developed the predetermined antibody response.

Double-blind assessment was maintained for 12 months, demonstrating no significant differences in cognition between antibody responders and the placebo group. In a small subset of patients, CSF tau levels were decreased in antibody responders but $A \beta$ levels were unchanged [Gilman et al. 2005]. Long-term follow up of treated patients and further analysis of autopsy data modified and moderated the negative impact of the first results, encouraging additional clinical attempts. Subsequent observations of AN1792vaccinated patients or transgenic models, and of brain tissue taken from mice and humans using a new tissue amyloid immunoreactive method suggested that antibodies against $A \beta$-related epitopes are capable of slowing down the progression of neuropathology in $\mathrm{AD}$. In a recent 4-year study, Hock and Nitsch followed 30 patients who received a primary and booster immunization in the first year after vaccination, providing further support for continuation of the investigation of antibody treatment in $\mathrm{AD}$ [Hock and Nitsch, 2005].

The occurrence of encephalitis led to the development of new vaccines, which lack the amino acid parts thought to be responsible for the T-cell response mediated encephalitis, but retain the residues $(4-10)$ required for antibodies to bind to $A \beta$. Most of these vaccines are now being tested in phase I and II trials: the CAD-106 trial led by Novartis/Cytos (Basel, Switzerland) and the V950 trial initiated by Merck (Whitehouse Station, NJ, USA) [Brody and Holtzman, 2008]. Additional antibodies under testing include ACC001 (Wyeth, New Jersey, USA, two phase II studies ongoing in the USA and Japan), MABT5102A (Genentech, San Francisco, 
California, USA, phase I completed), PF-04360365 (Pfizer, phase I completed), R1450 (Hoffman-LaRoche, Basel, Switzerland, phase I completed), GSK933776A (GlaxoSmithKline, London, UK, phase I completed) [ClinicalTrials. gov; Galimberti and Scarpini, 2011].

Given the adverse reactions of the active immunization and the variable antibody response to vaccines in older individuals, passive immunization directed against various domains of $A \beta$ emerged as an alternative immunotherapeutic strategy. A point of concern in these therapies is the occurrence of cerebral microhemorrhages. The underlying mechanism is probably related to vascular amyloid deposits (congophilic amyloid angiopathy), present in nearly all patients with AD. The need for vascular repair and regeneration during $\mathrm{A} \beta$ immunotherapy is another argument for early treatment and subtle clearance over a long period of time [Wilcock et al. 2007].

More advanced is the Elan/Wyeth trial of AAB001 monoclonal antibody (bapineuzumab), which entered phase III testing in 2007. This approach involves passive immunization with an A $\beta$ N-terminal directed, humanized monoclonal antibody. The murine version of this antibody binds to both soluble and aggregated $A \beta$. The multiple-dose phase II trial including 240 participants did not attain statistical significance on the primary efficacy endpoints in the whole study population. Some patients in the treatment group had a vasogenic edema, which is a serious side effect. However, in the subgroup of participants who did not have the apolipoprotein $\mathrm{E}$ (ApeE) $\varepsilon 4$ allele, clinically significant benefits were recorded in several scales and magnetic resonance imaging showed smaller loss of brain volume. Looking at the best result of different groupings, it seemed that a small subset of patients, the ApoE noncarriers who received the second lowest of the four doses six times, responded really well in 78 weeks. Therefore the phase III study was initiated in ApoE4 noncarriers with mild to moderate AD [Wisniewski and Konietzko, 2008].

The next most advanced trial to our knowledge is the Eli Lilly and Co. (Indianapolis, IN, USA) phase II trial of LY2062430 (solanezumab), which involves passive vaccination with an $A \beta$ central domain directed, humanized monoclonal antibody. Systemic administration of the closely related central domain mouse monoclonal antibody $\mathrm{m} 266$ rapidly improved behavioral performance and decreased plaque formation in preclinical studies. However, this antibody did not worsen intracerebral hemorrhage or vascular pathology in older APP transgenic mice. The phase II results have been reported and no safety concerns were raised; a phase III study is being conducted [Brody and Holtzman, 2008].

Finally, natural antiamyloid antibodies have been found in human intravenous immunoglobulins (IVIgs) obtained from the pooled plasma of healthy blood donors. In light of these observations, a phase I trial has been carried out in the USA. Eight patients with AD were treated with IVIg (Gammagard S/D immune globulin intravenous human) donated by Baxter Healthcare Corporation (Deerfield, IL, USA). Seven patients completed the study. After 6 months, cognitive function stopped declining in all seven patients and improved in six. In 2009, a phase III clinical trial involving more than 360 patients with $\mathrm{AD}$ was initiated and may provide conclusive evidence for the effect of IVIg as a treatment option for AD [Dodel et al. 2010].

Passive vaccination requires repeated infusions, which have a high cost. Therefore active vaccination is always taken into consideration.

Data from preclinical studies regarding mice suggest that novel immunotherapeutic strategies like DNA epitope vaccine [Qu et al. 2010], antibodies against the $\beta$-secretase cleavage site of the APP [Rakover et al. 2007] and mucosal vaccination [Hara et al. 2011] could be used as safe and effective methods for AD therapy. DNA epitope vaccines have received substantial interest because of the ease of selectively designing them to elicit specific immune responses. Mucosal vaccination is an alternative way to achieve humoral response. Its mechanism is based on the presence of lymphocytes in the mucosa of the nasal cavity and gastrointestinal tract. It produces primarily secretory IgA antibodies, but when the antigen is coadministered with adjuvants such as cholera toxin subunit B and heat labile Escherichia coli enterotoxin, substantial serum IgM titers can be achieved. It has a more limited humoral response with little or no cell-mediated immunity. The last developed mucosal immunotherapy for $\mathrm{AD}$ by nasal administration used a recombinant Sendai virus vector carrying $A \beta 1-43$ and mouse interleukin-10 cDNA. It induced good antibody responses to A $\beta$. When APP transgenic mice (Tg2576) received this vaccine once nasally, the $A \beta$ plaque 
Table 2. Disease-modifying treatments: modulation of tau deposition.

\begin{tabular}{lll}
\hline Interfering with tau deposition & $\begin{array}{l}\text { Interfering with tau } \\
\text { phosphorylation }\end{array}$ & Immunotherapy \\
\hline Methylene blue: phase II trial (+) & $\begin{array}{l}\text { Tau kinase inhibitors (lithium: } \\
\text { phase I trial (-) in AD (+) in } \mathrm{MCl} \text { ) }\end{array}$ & Vaccination: preclinical trials \\
\hline +, encouraging results; -, disappointing results. & \\
AD, Alzheimer's disease; $\mathrm{MCl}$, mild cognitive impairment.1
\end{tabular}

burden was significantly decreased 8 weeks later, without inducing inflammation in the brain. Tg2576 mice showed significant improvement in cognitive functions when examined 3 months after the vaccination [Hara et al. 2011].

\section{Disease-modifying treatments: modulation of tau deposition}

Drugs interfering with tau deposition. Multiple compounds have been identified through cell culture or in vitro screens as tau aggregation inhibitors (Table 2). A phenothiazine, methylene blue (MB) or methylthioninium chloride, has previously been used in humans and is currently being evaluated in AD trials. The problem with this drug is that urine is colored blue, resulting in a lack of blinding. However, promising results have emerged from a phase II clinical trial testing $\mathrm{MB}$ as a potential therapy for $\mathrm{AD}$, as improvements in cognitive function of patients with $\mathrm{AD}$ after 6 months of $\mathrm{MB}$ administration have been reported [Gura, 2008].

Drugs interfering with tau phosphorylation. The intriguing link between phosphorylation and tau pathology has provided the boost to examine the role of kinase inhibitors as potential therapeutics targeting tau. Kinases induce the hyperphosphorylation of tau [Yiannopoulou et al. 2009]. Despite the large number of tau phosphorylation sites and the ability of multiple kinases to phosporylate individual sites, glycogen synthase kinase 3 (GSK3 $\beta$ ) has emerged as a potential therapeutic target. The most studied compound able to inhibit GSK3 is lithium, but several other compounds are under development, including pyrazolopyrazines, pyrazolopyridines, the aminothiazole AR-A014418, and sodium valproate [Martinez and Perez, 2008]. In recent studies, the effect of short-term treatment on cognitive and biological outcomes in people with amnestic MCI was shown and supports the notion that lithium has disease-modifying elements with potential clinical implications in the prevention of AD [Forlenza et al. 2011].

Immunotherapy. Vaccination approaches targeting tau have been considered, but the development of a successful therapy is complicated because tau protein is intracellular [Galimberti and Scarpini, 2011].

\section{Disease-modifying treatments: modulation of inflammation and oxidative damage}

Anti-inflammatory drugs. Epidemiological evidence suggests that long-term use of NSAIDs protects against the development of AD. Despite this premise, prospective studies showed lack of efficacy [Aisen et al. 2002, 2003] or treatmentlimiting gastrointestinal toxicity [Rogers et al. 1993].

Molecules addressing oxidative damage. Potential antioxidants include mitoquinone, vitamin E, Ginkgo biloba, natural polyphenols such as green tea, wine, blueberries and curcumin, 13 fatty acids, folate, vitamin B6 and vitamin B12 supplementation. A trial to determine whether the reduction of homocysteine levels with highdose folate, vitamin B6 and vitamin B12 supplementation can slow the rate of cognitive decline in subjects with $\mathrm{AD}$ had no beneficial effect on the primary cognitive measure, the rate of change in ADAS-cog score over 18 months, or on any secondary measures, although the vitamin supplement regimen was effective in reducing homocysteine levels [Aisen et al. 2006]. Clinical trials with vitamin $\mathrm{E}$ and $\omega 3$ fatty acids did not show beneficial effects in patients with $\mathrm{AD}$ [Barten and Albright, 2008].

More recent data have revealed that tumor necrosis factor (TNF), one of the few gliotransmitters, has strikingly acute effects on synaptic physiology. These complex influences on neural health suggest that manipulation of this cytokine might have 
important impacts on diseases characterized by glial activation, cytokine-mediated neuroinflammation and synaptic dysfunction. Toward such manipulation in $\mathrm{AD}$, a 6-month study was conducted with 15 patients with probable AD who were treated weekly with perispinal injection of etanercept, an FDA-approved TNF inhibitor that is now widely used for the treatment of rheumatoid arthritis and other systemic diseases associated with inflammation. The results demonstrated that perispinal administration of etanercept could provide sustained improvement in cognitive function for patients with $\mathrm{AD}$. Additionally, the authors were impressed by the striking rapidity with which these improvements occurred in the study patients. Nevertheless, etanercept merits further study in RCTs [Griffin, 2008].

\section{Disease-modifying treatments:} additional approaches

Modulation of cholesterol and vascular-related risk factors. A link between hypercholesterolemia, cardiovascular diseases and AD has also been suggested. Additional vascular-related risk factors for $\mathrm{AD}$ include hypertension, atrial fibrillation, hyperhomocysteinemia, atherosclerosis and stroke [Hooijmans and Kiliaan, 2008]. Epidemiological studies have indicated that patients treated for cardiovascular disease with cholesterol-lowering therapy (statins) showed a decreased prevalence of AD [Jick et al. 2000]. The Lipitor's Effect in Alzheimer's Dementia (LEADe) study tested the hypothesis that a statin (atorvastatin $80 \mathrm{mg}$ daily) is beneficial to patients with mild to moderate $\mathrm{AD}$ receiving background therapy of donepezil $10 \mathrm{mg}$ daily. Despite a promising premise, there were no significant differences in the coprimary or secondary endpoints, although atorvastatin was generally well tolerated [Feldman et al. 2010].

Simvastatin metabolites are high-affinity 3-hydroxy-3-methyl-glutaryl-coenzyme A reductase inhibitors, reducing the quantity of mevalonic acid, a precursor of cholesterol. Cholesterol Lowering Agent (simvastatin) to Slow Progression (CLASP) of Alzheimer's Disease Study is an ongoing randomized, double-blind, placebo-controlled, parallel-assignment phase III trial that investigates the safety and effectiveness of simvastatin in slowing down the progression of AD. It has not yet published its results [McGuinness et al. 2010]. However, a randomized, doubleblind, placebo-controlled recent trial of simvastatin was conducted in individuals with mild to moderate AD and normal lipid levels. Simvastatin had no benefit on the progression of symptoms in individuals with mild to moderate $\mathrm{AD}$ despite significant lowering of cholesterol [Sano et al. 2011].

\section{Final remarks}

Currently available treatments for $\mathrm{AD}$ (donepezil, rivastigmine, galantamine and memantine) are symptomatic and do not decelerate or prevent the progression of the disease. However, these therapies demonstrate modest, but particularly consistent, benefit for cognition, global status and functional ability [Herrmann et al. 2011].

The search for disease-modifying interventions has focused largely on compounds targeting the A $\beta$ pathway. To date, many treatments targeting this pathway, such as tarenflurbil, tramiprosate and semagacestat, have been unsuccessful in demonstrating efficacy in the final clinical stages of testing [Gauthier et al. 2009; Imbimbo and Giardina, 2011].

However, colostrinin, scyllo-inositol, PBT2, avagacestat, etazolate and active and passive immunization methods, treatments also targeting the $\mathrm{A} \beta$ pathway, are being tested in advanced clinical trials.

At the same time, other possible neuronal mechanisms that seem to play important roles in the pathophysiology of this multifactorial disorder, such as tau deposition and hyperphosphorylation, neuroinflammation and oxidative stress, are being researched as promising therapeutic targets. Clinical trials with drugs interfering with tau deposition or phosphorylation (lithium) are ongoing [Martinez and Perez, 2008]. Clinical trials of potential antioxidants such as vitamin $\mathrm{E}$ and $\omega 3$ fatty acids did not show beneficial effects in patients with AD [Barten and Albright, 2008].

Etanercept, a TNF inhibitor that is now widely used for the treatment of systemic diseases associated with inflammation, provided sustained improvement in cognitive function in patients with mild to severe $\mathrm{AD}$ after perispinal administration in a 6-month, open-label pilot study. However, etanercept merits further study in RCTs [Griffin, 2008].

Modulation of cholesterol and vascular-related risk factors is an additional possible disease-modifying approach. CLASP is an ongoing phase III trial 
investigating the effectiveness of simvastatin in slowing down the progression of $\mathrm{AD}$ [McGuinness et al. 2010].

The development of disease-modifying drugs for $\mathrm{AD}$ is recognized as a worldwide necessity. These must presumably be drugs that will modify, either by stabilizing or slowing, the molecular pathological steps leading to neurodegeneration and finally dementia.

The required design of clinical trials to test this concept raises many questions regarding the study populations, the duration of trials, the necessary primary and secondary endpoints, including biomarkers [Vellas et al. 2007]. It has been recognized that, to modify $\mathrm{AD}$, which has recently been redefined to have presymptomatic and symptomatic phases, one must attempt to treat patients when neuronal dysfunction is far from full blown and largely irreversible [Dubois et al. 2010; Sperling et al. 2011].

The following considerations have emerged that should be taken into account when planning future clinical trials:

(1) The mechanisms underlying the pathogenesis of AD need to be thoroughly investigated before focusing on the development of novel disease-modifying compounds. Despite promising premises related to different pathogenic mechanisms, large phase III trials with potentially disease-modifying properties have failed to demonstrate any effect on cognition. It is of crucial importance to better understand the relationship between tau, $\mathrm{A} \beta$ and other factors to develop successful disease-modifying drugs [Galimberti and Scarpini, 2011].

(2) Treatments of AD appear effective only in certain phases of the disease. A few disease-modifying compounds have shown some benefits in mild but not moderate $\mathrm{AD}$ or even in MCI. Therapeutic trials should therefore be carried out as early as possible during the course of the disease, which requires the identification of more accurate tools for early diagnosis. New criteria for the diagnosis of AD have enlarged the window for the detection of the early stages of the disease and include biomarkers mechanistically related to AD pathology. Adoption of these early biomarkers in implementing design of future studies is highly desirable [Galimberti and Scarpini, 2011; Salomone et al. 2011].

(3) $\mathrm{AD}$ is heterogeneous in clinical presentation, underlying neuropathology and mixed causes (especially in late-onset AD). This fact is one more reason to improve our tools for detecting patients with amnestic MCI at high risk of converting to $\mathrm{AD}$ before the different full-blown clinical features of the disease appear. A major challenge will also be to identify subgroups with homogeneous biomarkers. At present, the focus in $\mathrm{AD}$ drug development is shifting from treatment to prevention [Salomone et al. 2011; Vellas et al. 2011].

(4) Indicators useful as surrogate outcome measures (surrogate biomarkers like magnetic resonance imaging, CSF tau and $\mathrm{A} \beta$, and amyloid positron emission tomography) should be identified to have substitutes for clinical endpoints (i.e. neuropsychological testing), tools able to predict clinical benefit or the opposite, and to demonstrate whether the drug has disease-modifying properties [Galimberti and Scarpini, 2011].

(5) Prolonged development times delay effective therapies from reaching patients in need. Several strategies are promising for answering the crucial question of, 'How much information is sufficient to proceed to phase III without excessive risk for failure?' Phase II proof of concept (POC) (IIa) and dose-finding (IIb) studies represent major challenges in drug development. Biomarkers, population enrichment with risk factors, clinical measures with greater sensitivity than standard trial instruments and adaptive dose-response designs might represent other strategies applicable to POC studies. All of these strategies are being considered as means of shortening phase IIb studies and creating a seamless interface with phase III. None of these strategies have been validated in a successful drug development program [Cummings, 2008b].

In conclusion, the new strategies seem to focus on examining the potential neuroprotective activity of disease-modifying drugs in the presymptomatic stages of AD, with the help of biomarkers that predict disease progression before development of overt dementia. 


\section{Funding}

This research received no specific grant from any funding agency in the public, commercial, or not-for-profit sectors.

\section{Conflict of interest statement}

Konstantina G. Yiannopoulou discloses no conflicts of interest. Sokratis G. Papageorgiou has received grants from Pfizer, Novartis and JanssenCilag for giving lectures in satellite symposia during neurological conferences.

\section{References}

Adlard, P. and Bush, A. (2006) Metals and Alzheimer's disease. F Alzheimers Dis 10: 145-163.

Aisen, P., Saumier, D., Briand, R., Laurin, J., Gervais, F., Tremblay, P. et al. (2006) A phase II study targeting amyloid-beta with 3APS in mild-to-moderate Alzheimer disease. Neurology 67: 1757-1763.

Aisen, P., Schafer, K., Grundman, M., Pfeiffer, E., Sano, M., Davis, K. et al. (2003) Effects of rofecoxib or naproxen vs placebo on Alzheimer's disease progression: a randomized controlled trial. $\mathcal{F} A M A$ 289: 2819-2826.

Aisen, P., Schmeidler, J. and Pasinetti, G. (2002) Randomized pilot study of nimesulide treatment in Alzheimer's disease. Neurology 58: 1050-1054.

Albert, J.S. (2009) Progress in the development of beta-secretase inhibitors for Alzheimer's disease. Prog Med Chem 48: 133-361.

Alfirevic, A., Mills, T., Carr, D., Barratt, B., Jawaid, A., Sherwood, J. et al. (2007) Tacrine-induced liver damage: an analysis of 19 candidate genes. Pharmacogenet Genomics 17: 1091-1100.

Almkvist, O., Darreh-Shori, T., Stefanova, E., Spiegel, R. and Nordberg, A. (2004) Preserved cognitive function after 12 months of treatment with rivastigmine in mild Alzheimer's disease in comparison with untreated $\mathrm{AD}$ and MCI patients. Eur F Neurol 11: 253-261.

Alva, G. and Cummings, J. (2008) Relative tolerability of Alzheimer's disease treatments. Psychiatry (Edgmont) 5: 27-36.

Ballard, C. and Corbett, A. (2010) Management of neuropsychiatric symptoms in people with dementia. CNS Drugs 24: 729-739.

Ballard, C., Corbett, A., Chitramohan, R. and Aarsland, D. (2009) Management of agitation and aggression associated with Alzheimer's disease: controversies and possible solutions. Curr Opin Psychiatry 22: 532-540.
Banerjee, S., Hellier, J., Dewey, M., Romeo, R., Ballard, C. and Baldwin, R. (2011) Sertraline or mirtazapine for depression in dementia (HTA-SADD): a randomised, multicentre, double-blind, placebocontrolled trial. Lancet 378: 403-411.

Barten, D. and Albright, C. (2008) Therapeutic strategies for Alzheimer's disease. Mol Neurobiol 37: 171-186.

Bartus, R., Dean, R., Beer, B. and Lippa, A. (1982)

The cholinergic hypothesis of geriatric memory dysfunction. Science 217: 408-414.

Bilikiewicz, A. and Gaus, W (2004). Colostrinin (a naturally occurring, proline-rich, polypeptide mixture) in the treatment of Alzheimer's disease. $\mathcal{F}$ Alzheimers Dis 6: 17-26.

Birks, J. (2006) Cholinesterase inhibitors for Alzheimer's disease. Cochrane Database Syst Rev (1): CD005593.

Blesa, R., Ballard, C., Orgogozo, R., Lane, J. and Thomas, S. (2007) Caregiver preference for rivastigmine patches versus capsules for the treatment of Alzheimer disease. Neurology 69: 23-28.

Brody, D. and Holtzman, D. (2008) Active and passive immunotherapy for neurodegenerative disorders. Annu Rev Neurosci 31: 175-193.

Cummings, J. (2008a) Optimizing phase II of drug development for disease-modifying compounds. Alzheimers Dement 4(1 Suppl. 1): 15-20.

Cummings, J. (2008b) The black book of Alzheimer's disease, part 1. Primary Psychiatry 15: 66-76.

Cummings, J. and Back, C. (1998) The cholinergic hypothesis of neuropsychiatric symptoms in Alzheimer's disease. Am F Geriatr Psychiatry 6: 64-78.

Cummings, J., Jones, R., Wilkinson, D., Lopez, O., Gauthier, S., Waldemar, G. et al. (2010) Effect of donepezil on cognition in severe Alzheimer's disease: a pooled data analysis. $\mathcal{F}$ Alzheimers Dis 21: 843-851.

Dodel, R., Neff, F., Noelker, C., Pul, R., Du, Y., Bacher, M. et al. (2010) Intravenous immunoglobulins as a treatment for Alzheimer's disease: rationale and current evidence. Drugs 70: 513-528.

Dubois, B., Feldman, H.H., Jacova, C., Cummings, J.L., Dekosky, S.T., Barberger-Gateau, P., et al. (2010) Revising the definition of Alzheimer's disease: a new lexicon. Lancet Neurol 9:1118-1127.

Farlow, M. (2002) A clinical overview of cholinesterase inhibitors in Alzheimer's disease. Int Psychogeriatr 14(Suppl. 1): 93-126.

Farlow, M., Alva, G., Meng, X. and Olin, J. (2010) A 25-week, open label trial investigating rivastigmine transdermal patches with concomitant memantine in mild-to-moderate Alzheimer's disease: a post hoc analysis. Curr Med Res Opin 26: 263-269. 
Farlow, M., Anand, R., Messina, J., Hartman, R. Jr and Veach, J. (2000) A 52-week study of the efficacy of rivastigmine in patients with mild to moderately severe Alzheimer's disease. Eur Neurol. 44: 236-241.

Faux, N., Ritchie, C., Gunn, A., Rembach, A., Tsatsanis, A., Bedo, J. et al. (2010) PBT2 rapidly improves cognition in Alzheimer's disease: additional phase II analyses. $\mathcal{F}$ Alzheimers Dis 20: 509-516.

Feldman, H., Doody, R., Kivipelto, M., Sparks, D., Waters, D., Jones, R. et al. (2010) Randomized controlled trial of atorvastatin in mild to moderate Alzheimer disease: LEADe. Neurology 74: 956-964.

Feldman, H., Schmitt, F. and Olin, J. Memantine MEM-MD-02 Study Group (2006) ADL in moderate-to-severe Alzheimer disease: an analysis of the treatment effects of memantine in patients receiving stable donepezil treatment. Alzheimer Dis Assoc Disord 20: 263-268.

Ferri, C., Prince, M., Brayne, C., Brodaty, H., Fratiglioni, L., Ganguli, M. et al. (2005) Global prevalence of dementia: a Delphi consensus study. Lancet 366: 2112-2117.

Forlenza, O., Diniz, B., Radanovic, M., Santos, F., Talib, L. and Gattaz, W. (2011) Disease-modifying properties of long-term lithium treatment for amnestic mild cognitive impairment: randomised controlled trial. Br F Psychiatry 198: 351-356.

Galimberti, D. and Scarpini, E. (2011) Diseasemodifying treatments for Alzheimer's disease. Ther Adv Neurol Disord 4: 203-216.

Gauthier, S., Aisen, P., Ferris, S., Saumier, D., Duong, A., Haine, D. et al. (2009) Effect of tramiprosate in patients with mild-to-moderate Alzheimer's disease: exploratory analyses of the MRI sub-group of the Alphase study. F Nutr Health Aging 13: 550-557.

Ghosh, A., Brindisi, M. and Tang, J. (2012) Developing $\beta$-secretase inhibitors for treatment of Alzheimer's disease. F Neurochem 120: 71-83.

Gill, S., Anderson, G., Fischer, H., Bell, C., Li, P., Normand, S. et al. (2009) Syncope and its consequences in patients with dementia receiving cholinesterase inhibitors: a population-based cohort study. Arch Intern Med 169: 867-873.

Gilman, S., Koller, M., Black, R., Jenkins, L., Griffith, S., Fox, N. et al. (2005) Clinical effects of Abeta immunization (AN1792) in patients with $\mathrm{AD}$ in an interrupted trial. Neurology 64: 1553-1562.

Golde, T. (2005) The Abeta hypothesis: leading us to rationally-designed therapeutic strategies for the treatment or prevention of Alzheimer disease.

Brain Pathol 15: 84-87.
Griffin, W. (2006) Inflammation and neurodegenerative diseases. Am $\mathcal{F}$ Clin Nutr 3(Suppl.): 470-474.

Griffin, W. (2008) Perispinal etanercept: potential as an Alzheimer therapeutic. F Neuroinflammation 5: 3.

Gura, T. (2008) Hope in Alzheimer's fight emerges from unexpected places. Nat Med 14: 894.

Hampel, H., Ewers, M., Bürger, K., Annas, P., Mörtberg, A., Bogstedt, A. et al. (2009) Lithium trial in Alzheimer's disease: a randomized, single-blind, placebo-controlled, multicenter 10 -week study. $\mathcal{F}$ Clin Psychiatry 70: 922-931.

Hansen, R., Gartlehner, G., Webb, A., Morgan, L., Moore, C. and Jonas, D. (2008) Efficacy and safety of donepezil, galantamine, and rivastigmine for the treatment of Alzheimer's disease: a systematic review and meta-analysis. Clin Interv Aging 3: 211-225.

Hara, H., Mouri, A., Yonemitsu, Y., Nabeshima, T. and Tabira, T. (2011) Mucosal immunotherapy in an Alzheimer mouse model by recombinant Sendai virus vector carrying A $\beta 1-43 / \mathrm{IL}-10$ cDNA. Vaccine 29: 7474-7482.

Herrmann, N., Chau, S., Kircanski, I. and Lanctôt, K. (2011) Current and emerging drug treatment options for Alzheimer's disease: a systematic review. Drugs 71: 2031-2065.

Hey, J., Koelsch, G., Bilcer, G., Jacobs, A., Tolar, M. and Tang, J. (2008) Single dose administration of the b-secretase inhibitor CTS21166 (ASP1720) reduces plasma A $\beta 40$ in human subjects. Presented by Dr Koelsch in the International Conference on Alzheimer's Disease (ICAD), Chicago, IL, USA, 26-31 July 2008. Available at: http:/www.alzforum. org/new/detail.asp?id=1790

Hock, C. and Nitsch, R. (2005) Clinical observations with AN1792 using TAPIR analyses. Neurodegener Dis 2: 273-276.

Hooijmans, C. and Kiliaan, A. (2008) Fatty acids, lipid metabolism and Alzheimer pathology. Eur $\mathcal{F}$ Pharmacol 585: 176-196.

Howard, R., McShane, R., Lindesay, J., Ritchie, C., Baldwin, A., Barber, R. et al. (2012) Donepezil and memantine for moderate-to-severe Alzheimer's disease. N Engl F Med 366: 893-903.

Imbimbo, B. and Giardina, G. (2011) $\gamma$-Secretase inhibitors and modulators for the treatment of Alzheimer's disease: disappointments and hopes. Curr Top Med Chem 11: 1555-1570.

Jick, H., Zornberg, G., Jick, S., Seshadri, S. and Drachman, D. (2000) Statins and the risk of dementia. Lancet 356: 1627-1631.

John, S., Thangapandian, S., Sakkiah, S. and Lee, K. (2011) Potent BACE-1 inhibitor design using pharmacophore modeling, in silico screening and 
molecular docking studies. BMC Bioinformatics 12(Suppl. 1): 28.

Maidment, I., Fox, C., Boustani, M., Rodriguez, J., Brown, R. and Katona, C. (2008) Efficacy of memantine on behavioral and psychological symptoms related to dementia: a systematic meta-analysis. Ann Pharmacother 42: 32-38.

Mancuso, C., Siciliano, R., Barone, E., Butterfield, D. and Preziosi, P. (2011) Pharmacologists and Alzheimer disease therapy: to boldly go where no scientist has gone before. Expert Opin Investig Drugs 20: 1243-1261.

Martinez, A. and Perez, D. (2008) GSK-3 inhibitors: a ray of hope for the treatment of Alzheimer's disease? f Alzheimers Dis 15: 181-191.

McGuinness, B., O’Hare, J., Craig, D., Bullock, R., Malouf, R. and Passmore, P. (2010) Statins for the treatment of dementia. Cochrane Database Syst Rev (8): CD007514.

McShane, R., Areosa Sastre, A. and Minakaran, N. (2006) Memantine for dementia. Cochrane Database Syst Rev (2): CD 003154.

Panza, F., Solfrizzi, V., Frisardi, V., Capurso, C., D'Introno, A., Colacicco, A.M. et al. (2009) Diseasemodifying approach to the treatment of Alzheimer's disease: from alpha-secretase activators to gammasecretase inhibitors and modulators. Drugs Aging 26: 537-555.

Qaseem, A., Snow, V., Cross, J., Forciea, M.Jr, Hopkins, R., Shekelle, P. Jr et al. American College of Physicians/American Academy of Family Physicians Panel on Dementia (2008) Current pharmacologic treatment of dementia: a clinical practice guideline from the American College of Physicians and the American Academy of Family Physicians. Ann Intern Med 148: 370-378.

Qu, B., Lambracht-Washington, D., Fu, M., Eagar, T., Stüve, O. and Rosenberg, R. (2010) Analysis of three plasmid systems for use in DNA A beta 42 immunization as therapy for Alzheimer's disease. Vaccine 28: 5280-5287.

Rakover, I., Arbel, M. and Solomon, B. (2007) Immunotherapy against APP beta-secretase cleavage site improves cognitive function and reduces neuroinflammation in $\mathrm{Tg} 2576$ mice without a significant effect on brain abeta levels. Neurodegener Dis 4: 392-402.

Reddy, V., Zhu, X., Perry, G. and Smith, M. (2009) Oxidative stress in diabetes and Alzheimer's disease. f Alzheimers Dis 16: 763-774.

Rogers, J., Kirby, L.C., Hempelman, S.R., Berry, D.L., McGeer, P.L., Kaszniak, A.W., et al. (1993) Clinical trial of indomethacin in Alzheimer's disease. Neurology 43:1609-1611.
Salloway, S., Sperling, R., Keren, R., Porsteinsson, A., van Dyck, C., Tariot, P. et al. ELND005-AD201 Investigators (2011) A phase 2 randomized trial of ELND005, scyllo-inositol, in mild to moderate Alzheimer disease. Neurology 77: 1253-1262.

Salomone, S., Caraci, F., Leggio, G., Fedotova, J. and Drago, F. (2011) New pharmacological strategies for treatment of Alzheimer's disease: focus on disease modifying drugs. Br f Clin Pharmacol 73: 504-517.

Sano, M., Bell, K., Galasko, D., Galvin, J., Thomas, R., van Dyck, C. et al. (2011) A randomized, doubleblind, placebo-controlled trial of simvastatin to treat Alzheimer disease. Neurology 77: 556-563.

Santa-Maria, I., Hernández, F., Del Rio, J., Moreno, F. and Avila, J. (2007) Tramiprosate, a drug of potential interest for the treatment of Alzheimer's disease, promotes an abnormal aggregation of tau. Mol Neurodegener 2: 17.

Siemers, E., Skinner, M., Dean, R., Gonzales, C., Satterwhite, J., Farlow, M. et al. (2005) Safety, tolerability, and changes in amyloid $\beta$ concentrations after administration of a $\gamma$-secretase inhibitor in volunteers. Clin Neuropharmacol 28: 126-132.

Silvestri, R. (2009) Boom in the development of non-peptidic beta-secretase (BACE1) inhibitors for the treatment of Alzheimer's disease. Med Res Rev 29: 295-338.

Sperling, R., Aisen, P., Beckett, L., Bennett, D., Craft, S., Fagan, A. et al. (2011) Toward defining the preclinical stages of Alzheimer's disease: recommendations from the National Institute on Aging-Alzheimer's Association workgroups on diagnostic guidelines for Alzheimer's disease. Alzheimers Dement 7: 280-292.

Stefani, M. and Liguri, G. (2009) Cholesterol in Alzheimer's disease: unresolved questions. Curr Alzheimer Res 6: 15-29.

Tariot, P., Farlow, M., Grossberg, G., Graham, S., McDonald, S. and Gergel, I. (2004) Memantine treatment in patients with moderate to severe Alzheimer disease already receiving donepezil: a randomized controlled trial. $\mathcal{F} A M A$ 291: 317-324.

Tong, G., Wang, J., Sverdlov, O., Huang, S., Slemmon, R., Croop, R. et al. (2012) Multicenter, randomized, double-blind, placebo-controlled, singleascending dose study of the oral $\gamma$-secretase inhibitor BMS-708163: tolerability profile, pharmacokinetic parameters, and pharmacodynamic markers. Clin Ther 34: 654-667.

Vassar, R. (2004) BACE1: the beta-secretase enzyme in Alzheimer's disease. $\mathcal{F}$ Mol Neurosci 23: 105-114.

Vellas, B., Aisen, P., Sampaio, C., Carrillo, M., Scheltens, P., Scherrer, B. et al. (2011) Prevention 
trials in Alzheimer's disease: an EU-US task force report. Prog Neurobiol 95: 594-600.

Vellas, B., Andrieu, S., Sampaio, C. and Wilcock, G. (2007) Disease-modifying trials in Alzheimer's disease: a European Task Force consensus. Lancet Neurol 6: 56-62.

Vellas, B., Sol, O., Snyder, P., Ousset, P., Haddad, R., Maurin, M. et al. EHT0202/002 study group (2011) EHT0202 in Alzheimer's disease: a 3-month, randomized, placebo-controlled, double-blind study. Curr Alzheimer Res 8: 203-212.

Wilcock, D., Jantzen, P., Li, Q., Morgan, D. and Gordon, M. (2007) Amyloid-beta vaccination, but not nitro-nonsteroidal anti-inflammatory drug treatment, increases vascular amyloid and microhemorrhage while both reduce parenchymal amyloid. Neuroscience 144: 950-960.
Wisniewski, T. and Konietzko, U. (2008) Amyloid- $\beta$ immunization for Alzheimer's disease. Lancet Neurol 7: 805-811.

Wong, G., Manfra, D., Poulet, F., Zhang, Q., Josien, H., Bara, T. et al. (2004) Chronic treatment with the $\gamma$-secretase inhibitor LY-411,575 inhibit $\beta$-amyloid peptide production and alters lymphopoiesis and intestinal cell differentiation. $\mathcal{F}$ Biol Chem 279: 12876-12882.

Yiannopoulou, K., Karydakis, K. and Sakka, P. (2009) Therapeutic targets in Alzheimer's disease. Primary Psychiatry 16: 29-36.

Zec, R. and Burkett, N. (2008) Non-pharmacological and pharmacological treatment of the cognitive and behavioral symptoms of Alzheimer disease. NeuroRehabilitation 23: 425-438.
Visit SAGE journals online http://tan.sagepub.com

(\$) SAGE journals 